\title{
Vitamin D supplementation of breastfed infants: a randomized dose-response trial
}

\author{
Ekhard E. Ziegler', Steven E. Nelson' ${ }^{1}$ and Janice M. Jeter ${ }^{1}$
}

BACKGROUND: Breastfed infants require supplementation with vitamin $\mathrm{D}(\mathrm{VD})$, but little is known about the necessary dose. This double blind trial evaluated four different doses of $\mathrm{VD}$.

METHODS: Exclusively breastfed infants $(N=213)$ were randomized at 1 mo to one of four doses, which they received through 9 mo while receiving no formula. The supplements provided daily 200 IU, 400 IU, 600 IU, or 800 IU of VD. The primary endpoint was plasma $25(\mathrm{OH}) \mathrm{D}$ level, and secondary outcomes were plasma parathyroid hormone and calcium, and illness incidence. The study was conducted during winter at $41^{\circ} \mathrm{N}$.

RESULTS: Most infants had low $(<50 \mathrm{nmol} / \mathrm{l}) 25(\mathrm{OH}) \mathrm{D}$ levels at $1 \mathrm{mo}$, but with supplementation levels rose. Overall, levels of $25(\mathrm{OH}) \mathrm{D}$ differed significantly in proportion to vD dose. There were no effects of vD on illness incidence or growth. Low levels were common, with $7.8 \%$ of levels being $<50 \mathrm{nmol} / \mathrm{l}$ and 15 infants having 2 to 4 low levels.

CONCLUSION: The four doses of $\mathrm{vD}$ produced different plasma levels of $25(\mathrm{OH}) \mathrm{D}$. The higher doses were somewhat more efficacious in maintaining VD sufficiency in breastfed infants. The findings support the recommended dose of $400 \mathrm{lU} / \mathrm{d}$, and stress the need to start supplementation at birth.

V itamin $\mathrm{D}(\mathrm{vD})$ is produced (cholecalciferol, $\mathrm{vD}_{3}$ ) in the skin upon exposure to uvB radiation. This endogenous production is strongly influenced by environmental factors, such as the extent of sun exposure, geographic latitude and season of the year, and by subject characteristics such as skin pigmentation $(1,2)$. Genetic factors also exert strong effects on the $\mathrm{vD}$ status (3). Exogenous (dietary) sources of $\mathrm{vD}_{3}$ and $\mathrm{vD}_{2}$ (ergocalciferol) can fully replace endogenously produced $\mathrm{vD}$, and thus, play an important role in situations where endogenous production of $\mathrm{vD}$ is limited or absent.

Infants are at risk of vD deficiency when endogenous production of $\mathrm{vD}$ is limited by dark skin pigmentation or by residence at a northern latitude. Breast milk provides native vitamins $\mathrm{D}_{3}$ and $\mathrm{D}_{2}$ as well as the respective 25 -hydroxylated compounds. But total antirachitic activity is typically $<100 \mathrm{IU} / \mathrm{l}$ and is often quite negligible (4-8). To ensure a daily intake of $400 \mathrm{IU} /$ day, the amount known to prevent rickets, it has for many years been recommended that breastfed infants receive $400 \mathrm{IU} /$ day of supplemental vD (9). By all accounts, this dose is effective in preventing rickets. In more recent years, the objective of supplementation has become the maintenance of $\mathrm{vD}$ status defined on the basis of plasma concentration of 25-hydroxy vD $(25(\mathrm{OH}) \mathrm{D})$. In spite of the paucity of data (10), the Institute of Medicine in 1997 established an adequate intake (AI) of $\mathrm{vD}$ for infants of $200 \mathrm{IU} /$ day (2). The American Academy of Pediatrics adopted the new AI, and in 2003, lowered the recommended supplementation dose for breastfed infants to 200 IU/day (11), only to revert back to $400 \mathrm{IU} /$ day in 2008 (ref. 12). In 2011, the Institute of Medicine raised the AI for infants back to $400 \mathrm{IU} /$ day (13). The present study was conceived to remedy the paucity of existing data, and had the objective of defining the relationship between $\mathrm{vD}$ intake and $\mathrm{vD}$ status of breastfed infants more precisely. Graded amounts of supplemental $\mathrm{vD}$ were provided from 1 to 9 mo while limiting as much as feasible the intake of vD from dietary sources. Study infants spent the key portion of the study in winter, thereby ensuring minimal endogenous production of $\mathrm{vD}$ at the study location (latitude $41^{\circ} \mathrm{N}$ ).

At the time the study was initiated, the recommended dose of supplemental vD was $200 \mathrm{IU} /$ day (11). In its original design, the study was to test 200,400 , and $600 \mathrm{IU} /$ day. The addition of a dose of $800 \mathrm{IU} /$ day was deemed necessary when a number of infants showed $25(\mathrm{OH}) \mathrm{D}$ levels $<50 \mathrm{nmol} / \mathrm{l}$ in spite of receiving $\mathrm{vD}$ supplements. The primary endpoint was plasma $25(\mathrm{OH}) \mathrm{D}$ concentration. Secondary outcomes were illness incidence and growth. Bone mineral content and measures of bone turnover were determined, but the findings are to be reported separately.

\section{RESULTS}

Two-hundred thirteen exclusively breastfed infants were enrolled at $1 \mathrm{mo}$ of age and were assigned at random to one of the four vD supplement doses. The flow of study subjects is shown in Figure 1. Infants who left the study did so mainly because the parents wished to introduce supplemental formula due to real or perceived insufficiency of the breast milk supply. Characteristics of infants who withdrew from the study did not 


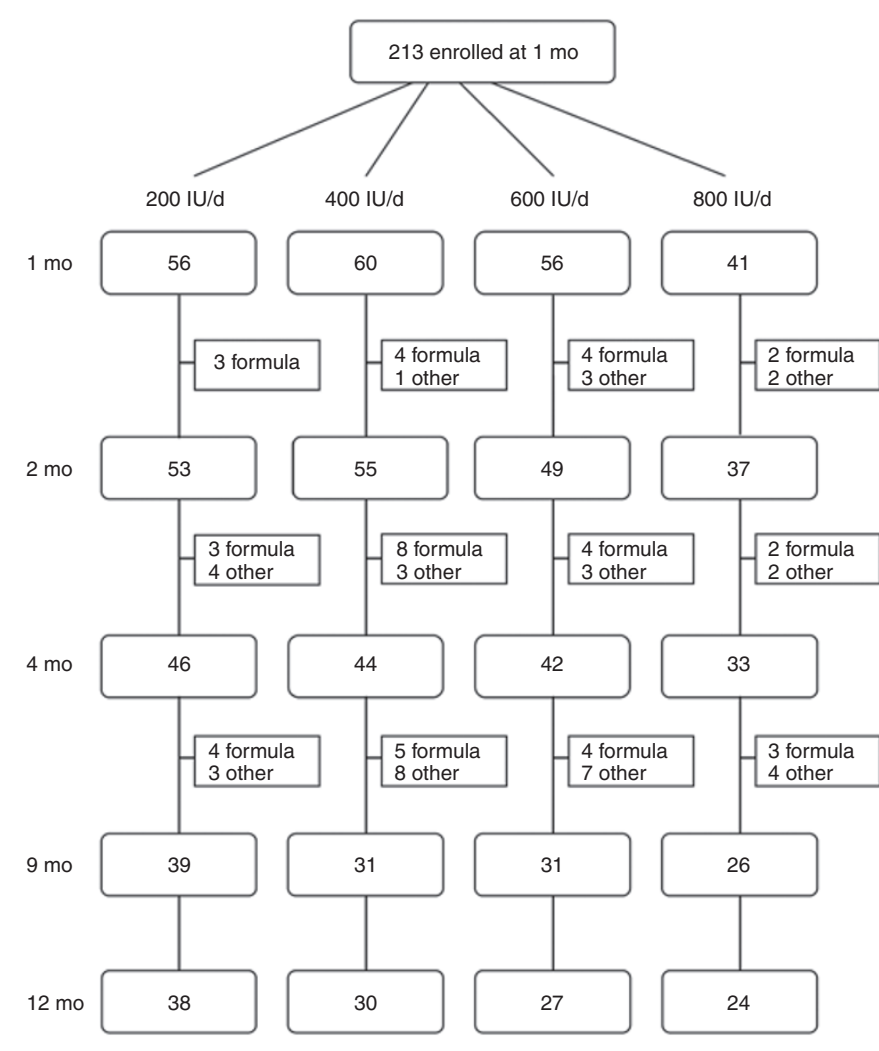

Figure 1. Flow of study subjects. Square boxes show number of subjects who left the study and the reason for it.

differ from those of infants who completed the study to 9 mo or to 12 mo. Beginning at $4 \mathrm{mo}$, infants were able to receive complementary foods, but could not receive supplemental formula until 9 mo. One infant (receiving $600 \mathrm{IU} /$ day) was withdrawn, because the parents felt the $\mathrm{vD}$ drops made the baby spit up. At 4 mo, 165 infants were in the study and of these 127 completed the intervention to 9 mo. Of the 119 infants followed to $12 \mathrm{mo}, 92$ were still breastfed. At the end of the winter (March 1 to mid-May) 142 infants were assessed.

At enrollment at $1 \mathrm{mo}$ of age and before the start of supplementation, infant plasma $25(\mathrm{OH}) \mathrm{D}$ levels averaged $41.0 \pm 19.7$ $\mathrm{nmol} / \mathrm{l}$, with $72 \%$ of levels $<50 \mathrm{nmol} / \mathrm{l}$. Maternal $25(\mathrm{OH})$ $\mathrm{D}$ concentrations $(N=181)$ obtained at the same time averaged $88.2 \pm 23.0 \mathrm{nmol} / \mathrm{l}$ and were, with only three exceptions, greater than $50 \mathrm{nmol} / \mathrm{l}$. Infant and maternal levels were highly correlated ( $r=0.427, P<0.0001)$. Every infant level was lower than the corresponding maternal level.

Plasma 25(OH)D concentrations are summarized in Table 1 and displayed in Figure 2. It is evident that average plasma $25(\mathrm{OH}) \mathrm{D}$ concentrations rose rapidly after the initiation of supplementation and kept rising until supplementation was discontinued at 9 mo. 25(OH)D levels increased significantly $(P<$ 0.001 ) with age (and duration of supplementation) and showed significant $(P<0.001)$ seasonal variation, with levels being higher in summer than in winter (ANOVA). At 12 mo (3 mo after discontinuation of supplementation), 25(OH)D levels were somewhat lower than at 9 mo and dose-dependent differences were no longer apparent. Multiple regression analysis showed that
25(OH)D concentration at 9 mo, besides being strongly dependent on vD dose $(P<0.001)$, was significantly influenced by the month of determination $(P=0.011)$ but not by birth weight or weight gain. A marked seasonal effect on $25(\mathrm{OH}) \mathrm{D}$ levels was also evident from the fact that low levels $(<50 \mathrm{nmol} / \mathrm{l})$ occurred almost exclusively from October to April (49 low levels) with only six low levels occurring between May and September. Multiple regression analysis also indicated that 9-mo 25(OH)D levels were significantly $(P=0.011)$ influenced by baseline levels at $1 \mathrm{mo}$, suggesting a genetic influence.

Determinations of calcium and parathyroid hormone (PTH) are summarized in Table 2. PTH levels did not show any effect of vD dose, but levels increased significantly $(P<0.027)$ with age. Calcium concentrations, on the other hand, increased overall significantly $(P=0.0002)$ with an increasing vD dose. Calcium also increased significantly $(P<0.0001)$ with age. Calcium concentrations $>12.0 \mathrm{mg} / 100 \mathrm{ml}$ were observed on one occasion with $200 \mathrm{IU} /$ day, on four occasions with $400 \mathrm{IU} /$ day, on three occasions with 600 IU/day and on three occasions with $800 \mathrm{IU} /$ day.

Laboratory data obtained at the end of winter, i.e., between March 1 and the middle of May, are summarized in Table 3. Infant ages averaged 7.1 mo. Multiple regression analysis showed that $25(\mathrm{OH}) \mathrm{D}$ concentrations at the end of the winter were strongly influenced by vD dose $(P<0.001)$ and were significantly influenced by baseline $25(\mathrm{OH}) \mathrm{D}$ concentration $(P=0.004)$ but not by other factors. Neither plasma calcium nor PTH at the end of the winter were affected by vD dose.

Growth data are summarized in Table 4. Gains in weight and in length were significantly greater in males than in females between 28 and $112 \mathrm{~d}(P<0.001)$ but not during subsequent age intervals. However, treatment-related differences in growth were very small and not statistically significant.

Consumption of vD supplements as determined by weighback of bottles is shown in Supplementary Table S1 online. When expressed as percent of target volume, there were no dose-related differences in consumption. Median individual consumption across ages was $103.4 \%$ of expected (interquartile range: $93.1-110.2 \%$ ) with minimum of $61.3 \%$ and maximum of $147.4 \%$. Thus, the average consumption was close to the target, but consumption by individual infants was consistently above or below the target amount. Consumption expressed as IU/day is summarized in Supplementary Table S1 online. One infant had zero consumption during one interval, whereas the highest recorded intake was 1,612 IU/day in an infant who was intended to receive $800 \mathrm{IU} /$ day. Not surprisingly, 25(OH)D levels were correlated with consumption of vD. Correlations tended to be weak on an age-specific basis for any given dosage, and strongest on an age-specific basis across dosages, where correlation coefficients ranged from 0.244 to $0.358(P<0.003)$.

A total of 185 episodes of illness were reported during the study. Illnesses occurred predominantly between October and April, and thus, coincided with low 25(OH)D levels. Yet there was no association between illness incidence and $25(\mathrm{OH}) \mathrm{D}$ levels. There was also no effect of vD dose on illness incidence 
Table 1. Plasma concentrations ( $\mathrm{nmol} / \mathrm{l}$ ) of $25(\mathrm{OH}) \mathrm{D}$ by supplement dose

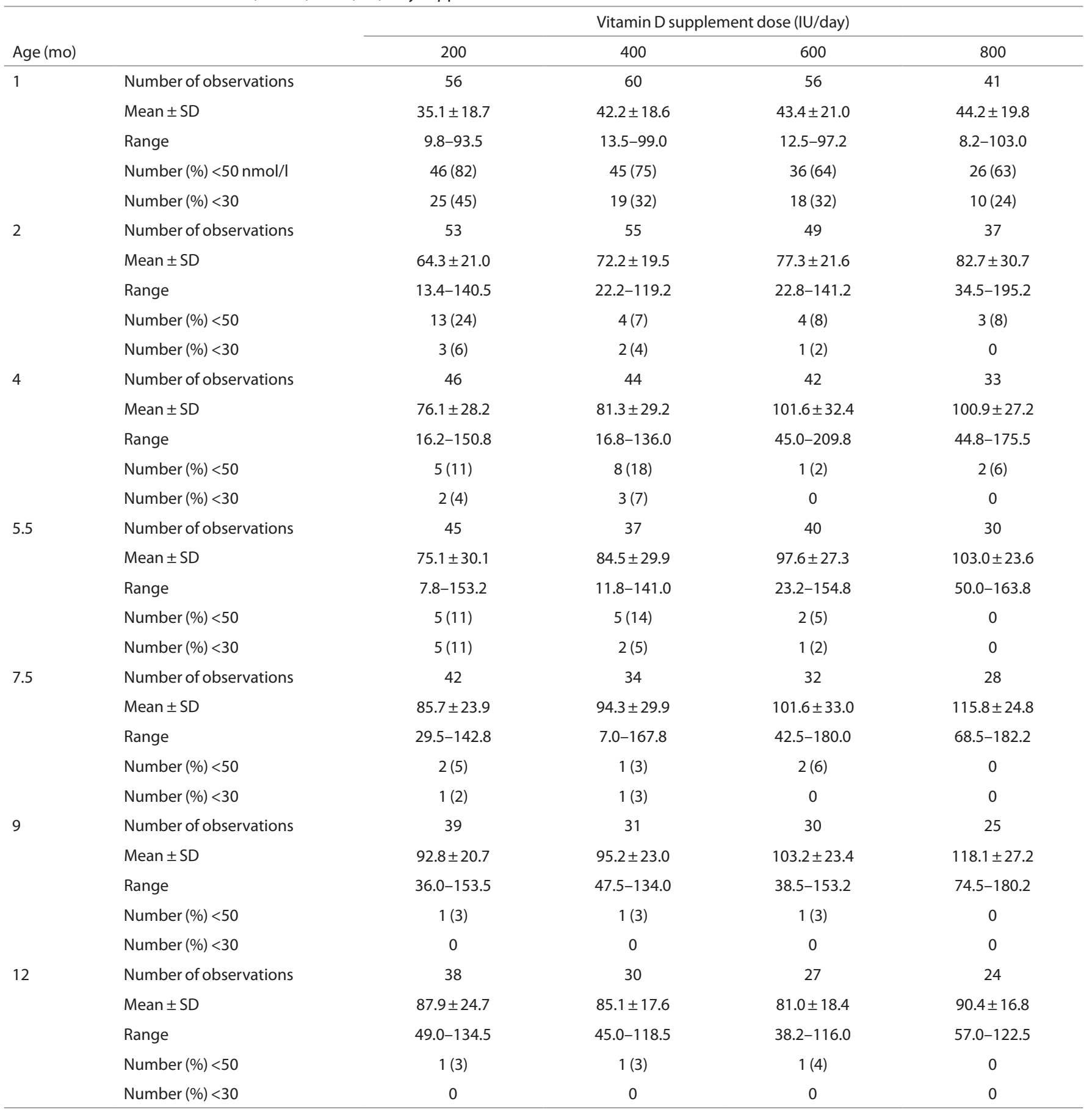

$\left(P=0.59, \chi^{2}\right.$ test $)$. We also compared months preceding and following a low $25(\mathrm{OH}) \mathrm{D}$ level (adjacent months) with all other months. The incidence was 0.058 illnesses per subject month during adjacent months and 0.111 illnesses per subject month during all other months; the difference was statistically significant $(P=0.002)$. This unexpected finding is perhaps explained by the fact that low 25(OH)D levels occurred predominantly between 2 and 4 mo, an age bracket in which infants tend to have fewer illnesses than later (illness incidence peaked around $7.5 \mathrm{mo}$ ).
Infants With Low 25(OH)D Levels

Low $25(\mathrm{OH}) \mathrm{D}$ levels were observed relatively frequently, while infants were receiving $\mathrm{vD}$ supplements (Supplementary Table S2 online). Overall, $7.8 \%$ of $25(\mathrm{OH}) \mathrm{D}$ levels were $<50$ $\mathrm{nmol} / \mathrm{l}$ and $19.4 \%$ of infants had at least one low level. Low levels were particularly common at 2 mo, i.e., 1 mo after the start of supplementation, when a total of 24 infants (12.4\%) still had low levels. Overall, low levels were more common with the two lower than with the two higher doses $(P=0.010)$, although even with $800 \mathrm{IU} /$ day, there were a total of 5 low 


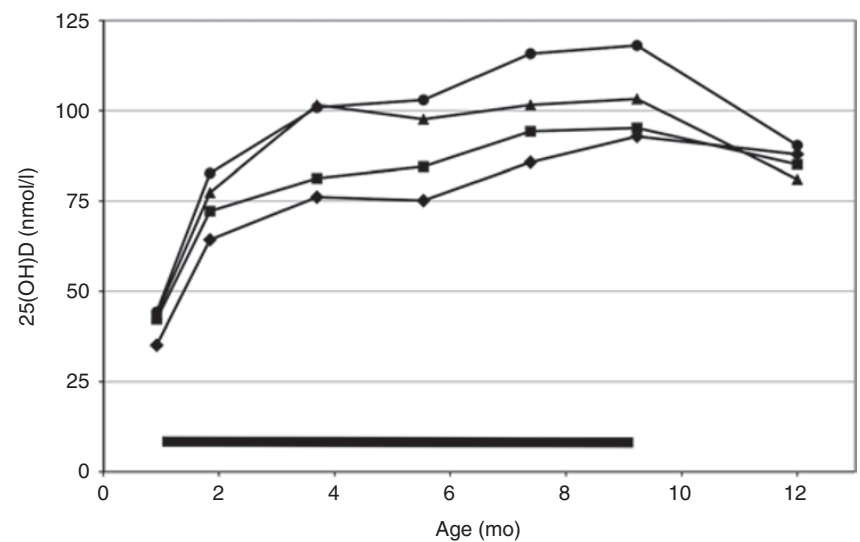

Figure 2. Mean plasma concentrations of $25(\mathrm{OH}) \mathrm{D}$ of infants receiving different doses of supplemental vD. Horizontal bar indicates period of vD supplementation. Black diamonds, $200 \mathrm{IU} / \mathrm{d}$; black squares, $400 \mathrm{IU} / \mathrm{d}$; black triangles, $600 \mathrm{IU} / \mathrm{d}$; black circles, $800 \mathrm{IU} / \mathrm{d}$. Concentrations differed overall significantly $(P<0.001)$ depending on supplement dose and increased significantly with age $(P<0.001)$.

Table 2. Plasma concentrations of PTH and calcium by supplement dose and age

\begin{tabular}{lccccc}
\hline & \multirow{4}{*}{$\begin{array}{c}\text { Age } \\
\end{array}$} & \multicolumn{5}{c}{ vD supplement (IU/day) } \\
\cline { 4 - 6 } & $(\mathrm{mo})$ & 200 & 400 & 600 & 800 \\
\hline PTH $^{\mathrm{a}}$ & 4 & $14.9 \pm 5.8$ & $15.7 \pm 7.6$ & $16.7 \pm 8.7$ & $18.0 \pm 6.9$ \\
$(\mathrm{ng} / \mathrm{l})$ & 5.5 & $15.7 \pm 7.6$ & $16.4 \pm 6.8$ & $17.0 \pm 7.3$ & $17.8 \pm 10.4$ \\
& 7.5 & $26.6 \pm 18.0$ & $17.9 \pm 9.3$ & $18.5 \pm 7.7$ & $19.1 \pm 11.5$ \\
& 9 & $22.4 \pm 14.4$ & $18.1 \pm 9.1$ & $21.8 \pm 10.9$ & $17.3 \pm 7.0$ \\
& 12 & $23.3 \pm 12.8$ & $20.9 \pm 14.3$ & $24.1 \pm 13.5$ & $21.8 \pm 8.2$ \\
Calcium $^{\mathrm{b}}$ & 2 & $9.97 \pm 0.58$ & $9.89 \pm 0.76$ & $9.94 \pm 0.54$ & $10.29 \pm 0.44$ \\
(mg/dl) & 4 & $10.17 \pm 0.68$ & $10.55 \pm 0.92$ & $10.09 \pm 0.90$ & $10.58 \pm 0.86$ \\
& 5.5 & $10.18 \pm 0.72$ & $10.42 \pm 0.75$ & $10.30 \pm 0.72$ & $10.50 \pm 0.83$ \\
& 7.5 & $10.19 \pm 0.61$ & $10.35 \pm 0.69$ & $10.07 \pm 0.82$ & $10.40 \pm 0.76$ \\
& 9 & $10.17 \pm 0.69$ & $10.54 \pm 0.65$ & $9.87 \pm 0.69$ & $10.60 \pm 0.73$
\end{tabular}

PTH, parathyroid hormone; $V D$, vitamin $D$.

aEffect of $v D$ not significant $(P=0.216)$, significant increase with age $(P=0.0267)$. beffect of vD significant $(P<0.0002)$, significant increase with age $(P \leq 0.0001)$.

levels. Supplementary Table S2 online also includes the number of levels below the cutoff of $30 \mathrm{nmol} / \mathrm{l}$ and the number of levels $<12.5 \mathrm{nmol} / \mathrm{l}$, which were observed in three infants.

Fifteen infants had more than one (i.e., 2-4) low 25(OH) $\mathrm{D}$ levels while receiving vD supplements. Except for one, the infants were Caucasian. As Supplementary Table S3 online shows, these "persistently low" infants entered the study with relatively low 25(OH)D levels as all but three infants had levels below the average 1-mo level of $41.0 \mathrm{nmol} / \mathrm{l}$. In the great majority of cases, low vD consumption did not explain low $25(\mathrm{OH}) \mathrm{D}$ levels. Serum concentrations of PTH and alkaline phosphatase of infants with 0 or 1 vs. 2 to 4 deficient $25(\mathrm{OH})$ $\mathrm{D}$ levels did not differ, nor were there differences with regard to growth and number of illnesses (data not shown). There were not any unusual plasma values in the three infants with $25(\mathrm{OH}) \mathrm{D}$ levels $<12.5 \mathrm{nmol} / \mathrm{l}$.
Table 3. Plasma concentrations at end of winter at a mean age of 7.1 mo

\begin{tabular}{lcccc}
\hline & \multicolumn{5}{c}{ Vitamin D supplement (IU/day) } \\
\cline { 2 - 5 } & 200 & 400 & 600 & 800 \\
\hline $\begin{array}{l}\text { Number of } \\
\text { observations } \\
25(\mathrm{OH}) \mathrm{D}(\mathrm{nmol} / \mathrm{l})\end{array}$ & 43 & 37 & 36 & 26 \\
Mean $\pm \mathrm{SD}$ & $76.4 \pm 25.8$ & $85.4 \pm 27.2$ & $95.3^{\mathrm{a}} \pm 25.6$ & $107.3^{\mathrm{a}} \pm 22.9$ \\
Range & $7.8-132.8$ & $11.8-141.0$ & $38.5-165.8$ & $74.5-182.2$ \\
$\begin{array}{l}\text { Number (\%) } \\
<50 \mathrm{nmol} / \mathrm{l}\end{array}$ & $5(12)$ & $3(8)$ & $2(5)$ & 0 \\
$\begin{array}{l}\text { Number }(\%) \\
<30 \mathrm{nmol} / \mathrm{l}\end{array}$ & $3(7)$ & $1(3)$ & 0 & 0 \\
PTH (ng/l) & $17.2 \pm 6.1$ & $17.2 \pm 7.6$ & $19.0 \pm 7.6$ & $16.8 \pm 5.6$ \\
$\begin{array}{l}\text { Calcium } \\
\text { (mg/dl) }\end{array}$ & $10.06 \pm 0.75$ & $10.45^{\mathrm{b}} \pm 0.76$ & $10.18 \pm 0.90$ & $10.44 \pm 0.78$ \\
\hline
\end{tabular}

avalues differ from value at $200 \mathrm{IU} /$ day at $P<0.001$. bValue differs from value at $200 \mathrm{IU} /$ day at $P=0.031$.

Two infants (subjects 17,194 and 17,215) had persistently very low 25(OH)D levels in spite of apparently consuming $106 \%$ and $100 \%$ of expected amounts of vD. Subject 17,194 had elevated PTH of 130,59 , and $68 \mathrm{ng} / \mathrm{l}$ at $4,5.5$, and $7.5 \mathrm{mo}$, respectively, with normal Ca levels of 9.6, 10.5, and 9.7 and normal alkaline phosphatase. In both infants treatment with $2,000 \mathrm{IU} /$ day of $\mathrm{vD}$ was recommended, whereupon $25(\mathrm{OH}) \mathrm{D}$ levels promptly rose (Supplementary Table S3 online).

\section{DISCUSSION}

The present study assessed the efficacy of four doses of supplemental $\mathrm{vD}$ with regard to the maintenance of vD sufficiency in breastfed infants. Breast milk often provides only small amounts of $\mathrm{vD}$, and the need for $\mathrm{vD}$ supplementation of breastfed infants is widely appreciated. The study did not include a placebo group because vD supplementation is recommended for all breastfed infants. One of the vD doses was $200 \mathrm{IU} /$ day, the recommended dose at the time of study initiation. The efficacy of vD supplementation was assessed under circumstances where endogenous production of $\mathrm{vD}$ could be assumed to be negligible (winter at latitude $41^{\circ} \mathrm{N}$ ) and while the intake of $\mathrm{vD}$ from the diet was presumed to be low (no formula). The prohibition against formula feeding was the reason why a number of infants left the study. Still, 127 mother-infant pairs (of 213 enrolled) completed the intervention from 1 to 9 mo.

The main finding of the study was that $\mathrm{vD}$ supplementation raised $25(\mathrm{OH}) \mathrm{D}$ levels in dose-dependent fashion. Plasma $25(\mathrm{OH}) \mathrm{D}$ concentrations during supplementation were comparable to those reported by others (14-16). Overall, the four doses of $\mathrm{vD}$ differed somewhat with regard to the maintenance of vD sufficiency. Based on the percentage of low 25(OH)D levels and the number of infants with persistently low levels, the two higher doses (600 and $800 \mathrm{IU} /$ day) were more efficacious than the lower doses (200 and 400 IU/day). However, both of the lower doses were adequate in maintaining vD status and appeared to be equally efficacious. Although mean plasma 
Table 4. Gain in weight and length. Differences related to dose of vD were not statistically significant (ANOVA)

\begin{tabular}{|c|c|c|c|c|c|c|}
\hline \multirow[b]{2}{*}{ Age period } & & & \multicolumn{4}{|c|}{ Vitamin D supplement (IU/day) } \\
\hline & & & 200 & 400 & 600 & 800 \\
\hline \multirow[t]{3}{*}{$1-4 \mathrm{mo}$} & Weight gain (g/day) & Females $^{a}$ & $22.6 \pm 3.6$ & $23.4 \pm 5.9$ & $22.8 \pm 9.4$ & $24.6 \pm 5.6$ \\
\hline & Length gain (mm/day) & Females $^{\mathrm{a}}$ & $0.93 \pm 0.11$ & $0.90 \pm 0.11$ & $0.91 \pm 0.13$ & $0.88 \pm 0.07$ \\
\hline & & Males & $1.02 \pm 0.11$ & $0.96 \pm 0.16$ & $0.97 \pm 0.13$ & $0.99 \pm 0.09$ \\
\hline \multirow{3}{*}{ 4-9 mo } & & Males & $12.5 \pm 3.70$ & $13.8 \pm 4.01$ & $11.7 \pm 2.75$ & $12.1 \pm 2.96$ \\
\hline & Length gain (mm/day) & Females & $0.48 \pm 0.07$ & $0.47 \pm 0.06$ & $0.48 \pm 0.08$ & $0.47 \pm 0.07$ \\
\hline & & Males & $0.48 \pm 0.08$ & $0.50 \pm 0.09$ & $0.48 \pm 0.08$ & $0.49 \pm 0.08$ \\
\hline
\end{tabular}

aFemales less than males at $P<0.001$.

concentrations of calcium increased significantly with increasing vD dose, there was no increase in the number of high $\mathrm{Ca}$ levels. There was thus no risk of hypercalcemia even with 800 IU/d. There was no effect of vD dose on plasma levels of PTH.

The majority of infants (72\%) entered the trial at 1 mo of age with low 25(OH)D levels which averaged $41.0 \pm 19.8$ $\mathrm{nmol} / \mathrm{l}$. These levels were very similar to the values reported by Wagner et al. (14), but were lower than the values reported by Gallo et al. (15). Infant levels were highly correlated with maternal levels 1 mo postpartum, as has been observed by others (17-19). Infant levels at 1 mo were less than one-half of maternal levels. Since at birth, infant levels were probably closer to maternal levels $(19,20)$, this suggests that infant levels had fallen appreciably since birth. The high prevalence of low levels at 1 mo strongly argues in favor of starting vD supplementation soon after birth (11).

Seasonal effects on $25(\mathrm{OH}) \mathrm{D}$ levels were apparent in our study, as was observed in infants before $(19,21)$, with levels significantly higher during summer than during winter. Evidence for a seasonal effect was also provided by the multiple regression analysis and by the fact that low levels occurred almost exclusively between October and April.

There was no discernible effect of $\mathrm{vD}$ dose on the incidence of illness. Not even in infants with repeated low 25(OH)D levels was there an increased incidence of illness. The fact that illness incidence in our breastfed infants was probably relatively low may have been contributing. Our study thus joins the long list of studies that failed to find an association between infectious illness and vD supplementation or status (22). It must be noted that our study did not include a placebo group, and was not powered to detect an effect on illness frequency. Failure to detect a protective effect is not tantamount to ruling out such an effect. Similar precautions must be applied to our finding that there was no effect of $\mathrm{vD}$ dose on growth.

An unexpected finding was the relatively high frequency with which low 25(OH)D levels were observed, and the fact that a substantial number of infants had multiple low levels while receiving vD supplementation. Gallo et al. (15) similarly reported failure on the part of some infants to reach vD sufficiency while receiving supplementation. It is not clear what explains these deficient levels. In some infants, the explanation was that they entered the trial in a deficient state. Noncompliance with supplement administration is an unlikely explanation since we monitored disappearance of study supplements and found little evidence of noncompliance. Some parents reported regurgitation of study drops, but this is an unlikely mechanism. Finally, the possibility that genetic factors are at least contributing must be considered (3). Consistent with the presence of genetic factors is the significant correlation between levels at 1 and $9 \mathrm{mo}$. Whatever is the explanation for low 25(OH)D levels, it is important and reassuring that this study was unable to detect any effects of deficient levels, whether single or repeated, on biochemical or other outcomes. There was not a hint of incipient rickets in any of the deficient infants. It may be concluded that a prerequisite for adverse effects vD deficiency to occur is that low 25(OH)D levels must exist for longer periods that was the case in our study.

Because the trial conditions were such that endogenous production of $\mathrm{vD}$ was minimal and intake of exogenous (dietary) $\mathrm{vD}$ was low, the findings could be extrapolated to other situations with low endogenous production of $\mathrm{vD}$, such as effective sun block use or residence in geographic areas with little sun exposure.

The study had a number of strengths. Besides being carried out in double-blind fashion, the study was designed to minimize endogenous production of vD. A further strength was that the intake of vD from nonstudy sources was minimized by prohibiting the feeding of formula. We are not aware of any other study involving substantial numbers of breastfed infants with very low vD intake from dietary sources. Another strength was that the consumption of supplemental $\mathrm{vD}$ was monitored and showed excellent compliance with study rules. Actual intake of supplemental vD seems not to have been determined previously.

A minor weakness was that the dose of $800 \mathrm{IU} / \mathrm{d}$ was added after the study was under way, albeit with maintenance of blinding and random assignment. The study had no placebo group because the need for vD supplementation of breastfed infants is firmly established and withholding of supplementation would have placed infants at undue risk of vD deficiency. 
In conclusion, the four different vD supplementation doses led to significantly different plasma levels of $25(\mathrm{OH}) \mathrm{D}$. At the start of the trial at $1 \mathrm{mo}$, the majority of infants had low 25(OH) D levels, which emphasizes the need to start supplementation soon after birth. Supplementation did not maintain plasma levels of $25(\mathrm{OH}) \mathrm{D}$ at $>50 \mathrm{nmol} / \mathrm{l}$ reliably, but the study was unable to identify any ill effects attributable to intermittently low levels. It was concluded that all four doses were effective in maintaining $\mathrm{vD}$ sufficiency in breastfed infants. The data support $400 \mathrm{IU} / \mathrm{d}$ as an $\mathrm{AI}$, and agree with the current recommendation of $400 \mathrm{IU} /$ day as a supplement for breastfed infants.

\section{METHODS}

\section{Study Design}

This was a prospective randomized trial conducted in double-blind fashion in breastfed infants. Its objective was to evaluate the effectiveness of different doses of vD supplements given from 1 to $9 \mathrm{mo}$. In its original design, it involved three doses of vD, 200, 400, and $600 \mathrm{IU} / \mathrm{d}$, and tested the hypothesis that one or more of the three vD doses would be efficacious in preventing vD deficiency, which was defined as occurrence of any $25(\mathrm{OH}) \mathrm{D}$ level $<50 \mathrm{nmol} / \mathrm{l}$ at any time while receiving supplementation. After enrollment of about one-half the planned number of infants, it became clear that deficient levels were more common than anticipated and that none of the doses completely prevented deficient levels from occurring. Therefore, an additional higher supplemental dose of $800 \mathrm{IU} /$ day was added, and the study hypothesis was modified to state that different doses of vD supplements are associated with different plasma levels of $25(\mathrm{OH}) \mathrm{D}$. The design modification was implemented with complete preservation of blinding. To ensure that the endogenous production of $\mathrm{vD}$ was minimal during the months preceding the main assessment, only infants born between June and November were enrolled. They would be between 5.5 and 9 mo old when assessed at the end of winter (March to May).

Randomization and blinding: Under the initial design, two letter codes were used for each of the three doses. Random sequences of the six codes (A-F) were generated using SAS proc plan. Randomization was stratified for gender and birth weight $(2,500-3,350 \mathrm{~g}$ vs. $>3,350 \mathrm{~g})$. At enrollment, infants were assigned to the next letter code on the list. Infants continued to receive the assigned supplement until 9 mo of age. All study personnel and parents were blinded to the identity of the supplements. Under the modified design, the new dose (800 IU/day) received three codes and the original doses each received two new codes. In this way, it could be expected that at the conclusion of the trial approximately equal numbers of infants would have received each of the four doses. New random sequences of nine codes ( $G-O)$ were generated. The identity of all codes was kept by the manufacturer of the supplements and was broken only after all study data had been gathered.

The study protocol and its modifications were reviewed and approved by the University of Iowa Institutional Review Board and parents provided written consent. The trial was registered with ClinicalTrials.gov under NCT00494104.

Infants were enrolled at 1 mo and visited the study center at monthly (28 d) intervals until 9 mo, and again at 12 mo. At each visit, an interval health history was obtained, and weight and length were measured. At predesignated ages, blood was obtained. At the enrollment visit at $28 \mathrm{~d}$ of age, maternal blood for determination of $25(\mathrm{OH})$ D was obtained. The primary study outcome was plasma $25(\mathrm{OH}) \mathrm{D}$ concentration, with concentrations of calcium and PTH as secondary outcomes. Although plasma bone-specific alkaline phosphatase, osteocalcin and C-terminal telopeptides (CTx) as well as bone mineral content by DEXA were also determined, findings are to be reported separately.

\section{Subjects}

Subjects were term infants (gestational age $\geq 37 \mathrm{wk}$ ) of either gender with birth weight $>2,500 \mathrm{~g}$ who were considered normal by their parents, physicians, and the investigators. The study team was not involved in providing health care to the subjects, but would answer questions by parents. Recruitment was through visits to local maternity units, advertisements, and word of mouth. Infants were exclusively breastfed at the time of enrollment, and remained so until 4 mo of age. Thereafter, parents were free to introduce complementary foods but not formula until completion of the intervention at 9 mo. Iron supplements were permitted but parents were asked to abstain from giving any vitamin supplements. Infants were born between August 2006 and September 2010. Six infants were African American, 5 were Native American, 9 were Hispanic, 2 were Asian, and 191 were Caucasian.

\section{Sample Size}

In its original design (September 2006), the study was to test the hypothesis that one or more of three $\mathrm{vD}$ doses $(200 \mathrm{IU} / \mathrm{d}, 400 \mathrm{IU} / \mathrm{d}$, and $600 \mathrm{IU} / \mathrm{d}$ ) was effective in preventing vD deficiency, which was defined as the occurrence of any plasma $25(\mathrm{OH}) \mathrm{D}$ concentration of $<50 \mathrm{nmol} / \mathrm{l}$ while receiving vD supplementation. We argued that for a regimen of $\mathrm{vD}$ supplementation to be considered effective, no more than $2 \%$ of subjects should develop vD deficiency. Thus, a $2 \%$ proportion of deficient infants represented the null hypothesis. We determined the number of subjects needed to keep the type I error at $5 \%$ and the type II error of falsely accepting the null hypothesis if in fact the true proportion of deficient subjects was 9 percent, at 20 percent (power 0.8). The number of subjects needed was 48 per group. With 48 subjects per group, we would be able to detect intergroup differences of $0.58 \mathrm{SD}$ with $\alpha$ of 0.05 and $\beta$ of 0.2 . Assuming an attrition rate of $20 \%$, we needed to enroll $3 \times 60=180$ infants at 1 mo of age. As mentioned, it appeared necessary to add a higher dose of vD (800 IU/day). With four doses, the number of infants needed to complete the study would have been 192 . However, the hypothesis underlying the sample size calculation was abandoned. Also, enrollment proceeded more slowly and attrition was higher than anticipated, primarily because infants were not permitted to receive supplemental formula until 9 mo. With 32 subjects per group, we would be able to detect intergroup differences of 0.72 SD with $\alpha$ of 0.05 and $\beta$ of 0.2 . The decision to terminate enrollment was made in October 2010, when it appeared that 32 infants per dose were likely to complete the intervention at 9 mo.

\section{Study Supplements}

The study vD supplements were prepared by UnitDrugCo in Centennial, CO, who also kept the code until the intervention was completed. Supplements were supplied in opaque bottles containing $50 \mathrm{ml}$ each. The source of $\mathrm{vD}_{3}$ (cholecalciferol) was initially fish oil and later became sheep's wool. Concentrations of vD were 400, 800, 1,200 , and $1,600 \mathrm{IU} / \mathrm{ml}$ so that the daily dose of $\mathrm{vD}$ was contained in a volume of $0.5 \mathrm{ml}$. The actual concentrations of vD were determined by Bio-Chem Consulting Services, Calgary, Alberta, Canada. Determined concentrations ranged from 90.3 to $123 \%$ of expected concentrations. Supplement bottles were code-labeled with the letters A through $\mathrm{O}$, with multiple letters used for each concentration. Parents were instructed to give $0.5 \mathrm{ml}$ each day directly into the infant's mouth, using the provided dropper marked at $0.5 \mathrm{ml}$. Supplement bottles were weighed before dispensing and when returned. The difference in weight was considered the amount consumed. Parents of two infants reported spillage of drops during two monthly periods and data for these periods were considered missing. Consumption of $\mathrm{vD}$ was calculated from the weight of supplement consumed, converted to volume (conversion $1 \mathrm{~g}=0.924 \mathrm{ml}$ ), and the analyzed $\mathrm{vD}$ concentration per $\mathrm{ml}$. Consumption was averaged for each subject across all age intervals and was expressed as percent of target volume and as IU/day.

\section{Procedures}

Infants were enrolled within $4 \mathrm{~d}$ of age $28 \mathrm{~d}$ ("1 mo") and were assigned to one of the doses. Infants visited the study center every $28 \mathrm{~d}( \pm 4$ d) until the completion of the intervention at $280 \mathrm{~d}(9 \mathrm{mo})$ of age. There was one follow-up visit at 12 mo of age. At each visit, weight and length were measured using standardized methods. Capillary blood was obtained at enrollment and at the visits at 2 mo (56 d), 
$4 \mathrm{mo}(112 \mathrm{~d}), 5.5 \mathrm{mo}(168 \mathrm{~d}), 7.5 \mathrm{mo}$ (224 d), $9 \mathrm{mo}$ (280 d), and $12 \mathrm{mo}$ (364 d) of age. At each visit, study supplement bottles were weighed for determination of consumption of supplement, and a fresh supply of weighed supplement was dispensed. An interval health history was obtained at each visit. Before each visit, the parents completed a questionnaire that recorded the frequency of breastfeeding, any feedings by bottle, and the type and amount of other foods on a recent typical day. During the visit, the questionnaire was checked for completeness and clarifications were obtained if necessary.

\section{Blood Collection}

Capillary blood was collected by heel stick using a disposable springloaded device (Tenderfoot, International Technidyne Corporation, Edison, NJ). Approximately $0.4 \mathrm{ml}$ of blood was collected into a heparin-treated tube and an additional $0.4 \mathrm{ml}$ was placed into an ethylenediaminetetraacetic acid-treated tube. Blood was centrifuged immediately and plasma separated from cells. At the $28-\mathrm{d}$ visit, venous blood ( $5 \mathrm{ml}$, ethylenediaminetetraacetic acid) was drawn from the infant's mother and was analyzed for $25(\mathrm{OH}) \mathrm{D}$.

\section{Laboratory Methods}

All determinations were performed on ethylenediaminetetraacetic acid-plasma except for calcium, which was determined on heparinplasma. Determinations of $25(\mathrm{OH}) \mathrm{D}$ were performed by Heartland Assays (Ames, IA) using an equilibrium radioimmunoassay (23). The assay had an intraassay CV of $8.2 \%$ and an interassay CV of $10.5 \%$ and does not cross-react with 3-epi-25(Oh)D (24). All other determinations were performed in-house. Calcium was determined colorimetrically using o-cresolphthalein complexone as reagent (Pointe Scientific, Canton, MI) with an intraassay CV of $1.5 \%$ and an interassay $\mathrm{CV}$ of $1.4 \%$. PTH was determined by an enzyme immunoassay using intact parathyroid hormone (ALPCO Diagnostics, Salem, NH) with an intra-assay CV of $3.0 \%$ and an interassay CV of $5.1 \%$.

\section{Statistical Methods}

The data were analyzed on an intention-to-treat basis. Descriptive statistics were generated for each dosage group at specific ages. Continuous data were analyzed cross-sectionally at specific ages by general linear models ANOVA with Tukey's tests, and longitudinally (2-9 mo) by a repeated measures mixed model ANOVA assuming compound symmetry using a maximum likelihood ratio test. Discrete data were assessed for independence in contingency tables using $\chi^{2}$ tests. Pearson correlation coefficients were used to evaluate relationships between various plasma parameters. Multivariate regression analysis was used to investigate the effect of $\mathrm{vD}$ dose and of possible other factors on measured vD concentration at the end of winter and at $9 \mathrm{mo}$ of age. Statistical significance was set at $P<0.05$.

Vitamin D deficiency was defined as a plasma $25(\mathrm{OH}) \mathrm{D}$ level $<50$ $\mathrm{nmol} / \mathrm{l}$. In addition, we used $<30 \mathrm{nmol} / \mathrm{l}(\leq 12 \mu \mathrm{g} / \mathrm{l})$ as well as $<12.5$ nmol/l as cut-offs.

\section{SUPPLEMENTARY MATERIAL}

Supplementary material is linked to the online version of the paper at http://www.nature.com/pr

\section{ACKNOWLEDGMENTS}

Laboratory analyses were performed by Charles Rebouché, $\mathrm{PhD}$, and Joyce Guese, CLA. Their work is gratefully acknowledged. Johannes Ledolter, PhD, provided valuable assistance with sample size estimation and statistical analysis; his help is gratefully acknowledged.

\section{STATEMENT OF FINANCIAL SUPPORT}

This study was supported by the National Institutes of Health, Bethesda, MD, grant HD048870 and registered at clinicaltrials.gov NCT00494104.

Disclosure: The authors have no conflicts of interest and no financial relationships relevant to this article to disclose.

\section{REFERENCES}

1. Holick MF. Vitamin D deficiency. N Engl J Med 2007;357:266-81.
2. Institute of Medicine. Dietary reference intakes for calcium, phosphorus, magnesium, vitamin D, and fluoride. Washington, DC: National Academies Press, 1997.

3. Wang TJ, Zhang F, Richards JB, et al. Common genetic determinants of vitamin D insufficiency: a genome-wide association study. Lancet 2010;376:180-8.

4. Specker BL, Tsang RC, Hollis BW. Effect of race and diet on human-milk vitamin D and 25-hydroxyvitamin D. Am J Dis Child 1985;139:1134-7.

5. Hollis BW, Pittard WB 3rd, Reinhardt TA. Relationships among vitamin $\mathrm{D}, 25$-hydroxyvitamin $\mathrm{D}$, and vitamin D-binding protein concentrations in the plasma and milk of human subjects. J Clin Endocrinol Metab 1986;62:41-4.

6. Ala-Houhala M, Koskinen T, Parviainen MT, Bidskotpi JK. 25-hydroxyvitamin D and vitamin D in human milk: effects of supplementation and season. Am J Clin Nutr 1988;48:1057-60.

7. Wagner CL, Hulsey TC, Fanning D, Ebeling M, Hollis BW. High-dose vitamin D3 supplementation in a cohort of breastfeeding mothers and their infants: a 6-month follow-up pilot study. Breastfeed Med 2006;1:59-70.

8. Dawodu A, Tsang RC. Maternal vitamin D status: effect on milk vitamin D content and vitamin D status of breastfeeding infants. Adv Nutr 2012;3:353-61.

9. Committee on Nutrition, American Academy of Pediatrics: The prophylactic requirement and the toxicity of vitamin D. Pediatrics 1963;31:17-30.

10. Specker BL, Ho ML, Oestreich A, et al. Prospective study of vitamin D supplementation and rickets in China. J Pediatr 1992;120:733-9.

11. Gartner LM, Greer FR; Section on Breastfeeding and Committee on Nutrition. American Academy of Pediatrics. Prevention of rickets and vitamin D deficiency: new guidelines for vitamin D intake. Pediatrics 2003;111(4 Pt 1):908-10.

12. Wagner CL, Greer FR; American Academy of Pediatrics Section on Breastfeeding; American Academy of Pediatrics Committee on Nutrition. Prevention of rickets and vitamin $\mathrm{D}$ deficiency in infants, children, and adolescents. Pediatrics 2008;122:1142-52.

13. Institute of Medicine. Dietary reference intakes for calcium and vitamin D. Washington, DC: The National Academies Press, 2011.

14. Wagner CL, Howard C, Hulsey TC, et al. Circulating 25-hydroxyvitamin $\mathrm{d}$ levels in fully breastfed infants on oral vitamin $\mathrm{d}$ supplementation. Int $\mathrm{J}$ Endocrinol 2010;2010:235035.

15. Gallo S, Comeau K, Vanstone C, et al. Effect of different dosages of oral vitamin D supplementation on vitamin D status in healthy, breastfed infants: a randomized trial. JAMA 2013;309:1785-92.

16. Abrams SA, Hawthorne KM, Rogers SP, Hicks PD, Carpenter TO. Effects of ethnicity and vitamin $\mathrm{D}$ supplementation on vitamin $\mathrm{D}$ status and changes in bone mineral content in infants. BMC Pediatr 2012;12:6.

17. Thomas SD, Fudge AN, Whiting M, Coates PS. The correlation between third-trimester maternal and newborn-serum 25-hydroxy-vitamin D in a selected South Australian group of newborn samples. BMJ Open 2011;1:e000236.

18. Novakovic B, Galati JC, Chen A, Morley R, Craig JM, Saffery R. Maternal vitamin $\mathrm{D}$ predominates over genetic factors in determining neonatal circulating vitamin D concentrations. Am J Clin Nutr 2012;96:188-95.

19. Streym SV, Moller UK, Rejnmark L, Heickendorff L, Mosekilde L, Vestergaard P. Maternal and infant vitamin D status during the first 9 months of infant life - a cohort study. Eur J Clin Nutr 2013;67:1022-8.

20. Merewood A, Mehta SD, Grossman X, et al. Widespread vitamin D deficiency in urban Massachusetts newborns and their mothers. Pediatrics 2010;125:640-7.

21. Ziegler EE, Hollis BW, Nelson SE, Jeter JM. Vitamin D deficiency in breastfed infants in Iowa. Pediatrics 2006;118:603-10.

22. Camargo CA Jr, Manson JE. Vitamin D supplementation and risk of infectious disease: no easy answers. Am J Clin Nutr 2014;99:3-4.

23. Hollis BW, Kamerud JQ, Selvaag SR, Lorenz JD, Napoli JL. Determination of vitamin D status by radioimmunoassay with an 125I-labeled tracer. Clin Chem 1993;39:529-33.

24. Carter GD. 25-hydroxyvitamin D: a difficult analyte. Clin Chem 2012;58:486-8. 